\title{
A IMPORTÂNCIA DA DOMÓTICA PARA A SUSTENTABILIDADE DAS CIDADES 1
}

\author{
THE IMPORTANCE OF THE DOMOTICS FOR THE SUSTAINABILITY OF \\ CITIES
}

\author{
Ricardo Gil Domingues \\ Universidade Federal do Rio de Janeiro, PEU/POLI/UFRJ \\ gilmeth@poli.ufri.br \\ Armando Carlos de Pina Filho \\ Universidade Federal do Rio de Janeiro, PEU/POLI/UFRJ \\ armando@poli.ufri.br
}

\begin{abstract}
Resumo
O advento de novas tecnologias, dos computadores e da Internet, tem proporcionado importantes mudanças no mundo moderno, principalmente com relação aos aspectos tecnológicos e sociais. Particularmente, a habitação tem atraído grande interesse das comunidades técnicas e científicas, objetivando o emprego de uma nova ciência para promover o bem-estar social, conforto e qualidade de vida: a Domótica. Basicamente, essa ciência consiste na automação doméstica das habitações (casa, escritório ou residência), fazendo uso da junção multidisciplinar de muitas especialidades, como eletricidade, mecânica, psicologia, telecomunicações, informática e medicina, na tradução de qualidade de vida para seus moradores e usuários, gerando conforto, segurança, lazer, comunicação e racionalização de energia. Todos esses benefícios são obtidos com utilização eficaz dos recursos e com sustentabilidade. Dessa forma, o presente trabalho resulta de uma série de discussões e reflexões sobre a questão da Domótica na habitação das cidades, na procura de maior sustentabilidade social, econômica e ambiental. Pretende-se mostrar, de fato, que nos dias atuais e com o atual estilo de vida urbano, as cidades tendem a se tornar cada vez mais dependentes das novas tecnologias aplicadas à habitação.
\end{abstract}

Palavras-chave: Habitação. Domótica. Sustentabilidade.

\begin{abstract}
The advent of new technologies, computers and the Internet, has provided significant changes in the modern world, mainly with respect to technological and social aspects. Particularly, habitation has attracted great interest of technical communities, and scientific, aiming the use of a new science to promote mainly the social well-being, comfort and quality of life: Domotics. Basically, this science consists in the home automation of dwellings (home, office or residence), making use of the junction of many multidisciplinary specialties such as: electricity, mechanics, psychology, telecommunications, computing and medicine, in the translation of quality of life for its residents and users, generating comfort, security, leisure, communication, and rationalization of energy. All these benefits are obtained with efficient usage of resources and with sustainability. Thus, this work is the result of a series of discussions and reflections on the question of Domotics in the habitation, in the searching of greater social, economic and environmental sustainability. We intend show, in fact, that in the current days and with the current urban lifestyle, the cities tend to become increasingly more dependent on new technologies applied to housing.
\end{abstract}

Keywords: Habitation. Domotics. Sustainability.

\footnotetext{
${ }^{1}$ DOMINGUES, R. G.; PINA FILHO, A. C. de. A Importância da Domótica para a Sustentabilidade das Cidades. In: ENCONTRO BRASILEIRO DE TECNOLOGIA DE INFORMAÇÃO E COMUNICAÇÃO NA CONSTRUÇÃO, 7., 2015, Recife. Anais... Porto Alegre: ANTAC, 2015.
} 


\section{7}

\section{TIC2015}

\section{INTRODUÇÃO}

A tecnologia modificou de forma irreversível a maneira como a sociedade atual se relaciona, como a população trabalha e de que forma ela ocupa o seu tempo em casa. $\mathrm{O}$ acesso à informação e as facilidades de se trabalhar na residência ilustram bem como a sociedade tem evoluído ao longo da última década, em particular, nos grandes centros urbanos. Podese dizer que a natureza e a função da casa estão mudando consideravelmente, o que proporciona transformações na medida em que atinge as relações sociais, a interação familiar, a vida diária e a estrutura da cidade. A rapidez com que as tecnologias da informação e as automações domésticas estão sendo introduzidas na vida da sociedade está mudando o conceito de habitação, o que ela pode proporcionar, e de que forma ela terá de evoluir para atender as necessidades atuais da população. $O$ que até muito pouco tempo era conveniente, agora não passa do básico e as tendências atuais em termos tecnológicos e sociais fazem com que seja reavaliado o conceito de "habitar".

As crescentes exigências da sociedade com relação ao conforto para a habitação se somam com a possibilidade de melhorar o desempenho de questões como a da sustentabilidade ambiental onde a gestão de recursos naturais, principalmente a água e a energia, se fazem presentes na diminuição dos desperdícios e consequentemente numa diminuição de custos financeiros.

Outro fator relevante nessa integração habitação - tecnologia é a promoção da sustentabilidade social, onde a capacidade de acesso a serviços aumenta, proporcionando uma maior participação da sociedade, assim como uma maior autonomia na execução de tarefas domésticas, principalmente por parte de grupos sociais com alguma deficiência ou incapacidade, e idosos (ELOY et al., 2010).

A adaptação de novas funções na habitação obriga a novas abordagens no projeto do espaço doméstico e a um novo desenho, com a possibilidade de formar ambientes multifuncionais onde se possa, por exemplo, trabalhar em casa através de teletrabalho (trabalho à distância) ou mesmo auxiliar no tratamento de pessoas enfermas. A necessidade de espaço físico, juntamente com a flexibilidade e integração dos espaços habitacionais provocam também a necessidade da existência de soluções de construção e arquitetura para alocação física da infraestrutura a ser incorporada, além de uma compatibilidade das redes de serviço das cidades através de novas articulações.

O mercado imobiliário está começando a se adequar a essa nova realidade da Domótica, onde o edifício inteligente já aparece, otimizando a oferta de serviços do condomínio e conjugando o mesmo com a residência. Essa inovação está cada vez mais se traduzindo num diferencial na opção de compra de novas habitações, sem falar na valorização das atuais através de suas adaptações.

Logo, o presente trabalho procura avaliar o contexto apresentado, levando em consideração uma série de discussões e reflexões sobre a questão da Domótica na habitação das cidades, na procura de maior sustentabilidade social, econômica e ambiental. Pretende-se mostrar, de fato, que nos dias atuais e com o atual estilo de vida urbano, as cidades tendem a se tornar cada vez mais dependentes das novas tecnologias aplicadas à habitação.

\section{DOMÓTICA}

Pode-se dizer que a Domótica é uma evolução da automação residencial. O termo "Domótica" resulta da junção da palavra do latim "Domus" (casa) com "Robota", do tcheco (servo), sugerindo controle automatizado de algo na casa. Ela consiste, basicamente, na promoção da gestão dos recursos habitacionais de uma habitação (casa, escritório ou residência), fazendo uso da junção multidisciplinar de muitas especialidades, como eletricidade, mecânica, psicologia, telecomunicações, informática e medicina, na tradução 
de qualidade de vida para seus moradores e usuários, gerando conforto, segurança, lazer, comunicação e racionalização de energia. Tudo isso com utilização eficaz dos recursos e com sustentabilidade.

O objetivo maior da Domótica é simplificar a vida do habitante dentro do domicílio, facilitando a sua interação com o ambiente. A expectativa é que os equipamentos de uma residência, principalmente os aparelhos eletrodomésticos, sejam conectados em rede, de forma que possam ser gerenciados através de comandos e monitoramentos remotos e interativos, tanto internos quanto externos à habitação. Nesses ambientes, a computação ubíqua ou "invisível" é uma ferramenta bastante utilizada tanto na assistência ao usuário quanto na análise do funcionamento das mesmas. A proposta da computação ubíqua atual situa os habitantes num ambiente onde eles viverão rodeados de interfaces e dispositivos eletrônicos imperceptíveis, em constante interação com o mundo virtual. A ideia final é a de que computadores serão instalados em paredes, móveis, roupas e objetos de forma natural e espontânea, com características de tomada de decisão, aprendizagem e interação proativa, onde a antecipação da ação se torna uma opção real (YUSUPOV \& RONZHIN, 2010).

Logicamente, para se alcançar um ambiente inteligente, onde haja uma integração adequada entre os diversos sistemas domóticos e a habitação, foi necessária a realização de várias pesquisas, buscando-se uma evolução não apenas tecnológica, mas também de costumes sociais. Um histórico dessa evolução, da utilização da Domótica na habitação, pode ser encontrado em trabalhos como Forty (1986), Aldrich (2003), e Satpathy (2006).

\section{ASPECTOS SOCIAIS DA DOMÓTICA NO CONTEXTO HABITACIONAL}

Tem-se assistido no mundo a uma crescente transformação de ideias e hábitos, graças à globalização, juntamente com uma procura maior da sociedade por formas de se atingir a sustentabilidade social, econômica e ambiental. Segundo Domingues (2013), o contexto habitacional também passa por mudanças que a própria sociedade tem imposto na habitação através da alteração dos limites funcionais dos novos espaços "digitais" criados pelo uso da Domótica. Os ambientes de uma residência começam a não ter "regras" de uso e sua utilização acaba ficando dependente dos recursos disponibilizados no mesmo, bem como do comportamento social.

De acordo com Guerra (2000) apud Eloy (2010), a forma como as pessoas vivem e coabitam vem sendo moldada de acordo com vários fatores. A situação econômica é um dos quesitos mais importantes. A transformação da casa acaba sendo envolvida pela renda e pelo emprego, promovendo a. aquisição de bens e serviços, bem como a Educação, a qual é o combustível da promoção social.

O crescimento da utilização dos sistemas de comunicação em conjunto, (telefonia móvel e Internet) tem possibilitado conexão, monitoramento, controle e atualização de informações de forma bem acessível, alterando a forma como as pessoas trabalham, estudam e se relacionam.

No que diz respeito à família, existem transformações no comportamento sociocultural (LARCHER, 2005), onde se vê uma distinção menos formal entre os elementos do casal, e a maior permissividade para as crianças. A família também está diminuindo, com a redução do número de filhos e, consequentemente, a redução da dimensão familiar total. Outra característica envolvendo a família é a jornada de trabalho do casal, que tem que empregar uma babá ou deixar algum familiar tomando conta das crianças.

O número de pessoas que moram sozinhas está aumentando, juntamente com uma tendência a diminuição de vida coletiva e sua substituição pela autonomia e por valores de conquista pessoal tem uma característica bastante comum. 
O aumento da qualidade de vida tem expandido o tempo de duração das pessoas. $\mathrm{O}$ forte envelhecimento da população constitui um dos aspectos mais marcantes da evolução demográfica recente. Tomando o Brasil como referência, segundo dados do UN Department of Economic and Social Affairs, Population Division (2010), a previsão do percentual de pessoas com mais de 65 anos, em 2050, terá aumentado $221 \%$ em relação a 2010, onde a proporção em relação à população total brasileira passará de $8 \%$ para $22,5 \%$.

O número de pessoas com alguma deficiência também tem aumentado em alguns países. Tomando o Brasil como referência, segundo o Instituto Brasileiro de Geografia e Estatística (IBGE - Censo 2010), esse grupo possui um valor expressivo, chegando a $24 \%$ da população total em 2010. Estima-se que aproximadamente 15 milhões de brasileiros (8\%) são dependentes de cuidados diários, como cegueira, demência e paraplegia e que 4 milhões de habitantes $(2,1 \%)$ tem deficiência motora grande ou total.

\section{SUSTENTABILIDADE SOCIAL}

A Habitação tem tido necessidade de se adaptar aos novos padrões de ocupação da sociedade, onde a tecnologia tem aparecido para facilitar esse processo com o uso da Domótica. As metodologias decorrentes desse uso se traduzem sobretudo em economia de tempo na execução de tarefas, que o usuário poderia realizar em atividades mais interessantes como, por exemplo, ficar com os familiares.

No que diz respeito à aceitação, os grupos sociais constituídos por pessoas mais jovens e ativas tendem a receber bem as novidades tecnológicas. Com relação ao grupo de pessoas de mais idade, eles têm certa resistência, porque precisam ver claramente os benefícios antes de tomar a decisão de adquirir a tecnologia. É um grupo que não tem tanto dinheiro para "arriscar" na compra de equipamentos para depois não usar. Possuem uma posição pragmática, ou seja, não possuem muita expectativa. Esse grupo, porém, dependendo da condição cultural e econômica do país, já está sendo substituído pelas novas gerações que tiveram mais contato com as inovações tecnológicas e se familiarizam mais com as novas interfaces, as quais vêm se tornando cada vez mais amigáveis.

A vantagem da Domótica para os grupos formados por idosos e incapacitados consiste na promoção da vida independente, permitindo que os mesmos possam continuar morando em suas casas, com auxílio de tecnologias que tragam conforto e ajuda na realização de tarefas, proporcionando um sentimento de poder e autoestima. A inclusão social também é proporcionada, traduzindo-se em maior participação na comunidade através das redes sociais. A carga psicológica envolvendo a família, nesses grupos, bem como os serviços de assistência pessoal, podem ser reduzidos com o uso de equipamentos como os de teleajuda (health care). Eles proveem o monitoramento dos sinais biológicos através de dispositivos embarcados, seja em pulseiras ou roupas, significando um recurso prático para o caso de emergências, onde o usuário, ao ativar o aparelho, aciona um serviço de pronto atendimento.

Entre os recursos proporcionados pela Domótica, que convergem para a sustentabilidade social, pode-se citar o teletrabalho, a teleconferência, a telemedicina e a tele-educação, entre outros.

O teletrabalho (home office) é uma atividade que tem permitido ao usuário trabalhar em casa, conciliando a vida profissional e a familiar, com possibilidades de flexibilização do horário de trabalho e ganho de tempo e dinheiro, evitando-se deslocamentos casa-trabalhocasa. Como essa atividade faz uso de redes de dados e informação, o trabalhador não precisa se concentrar nos grandes centros urbanos, favorecendo o meio rural. Com isso, evita-se a exclusão social, integrando no mercado de trabalho pessoas que não podem sair de casa, a exemplo de idosos e portadores de alguma incapacidade. Como consequências, 
o teletrabalho pode levar ao isolamento social, à degradação da vida familiar e à exploração do trabalho.

A teleconferência ou videoconferência é um meio de comunicação, onde os sinais de áudio e vídeo são disponibilizados em tempo real, podendo ser uma solução para a questão de contatos entre pessoas distantes. Isto pode permitir que, alguém possa estar, por exemplo, tomando café com outra pessoa especial morando do outro lado do mundo, na sua sala de jantar, como se estivessem juntos, permitindo ampla conectividade.

A telemedicina ou medicina à distância é um tipo de teleconferência onde o paciente é atendido pelo médico. É mais um recurso que pode ser futuramente oferecido de forma básica, principalmente para os usuários que se encontram longe dos grandes centros urbanos. Por enquanto, em alguns países, está sendo testada a visita virtual, onde o médico consegue receber os dados biológicos do usuário via rede e, após um exame dirigido, fazer uma pré-avaliação ou mesmo uma prevenção de determinada anormalidade em relação à saúde do paciente. É uma tendência, porém existem usuários, geralmente da área rural, que ainda preferem ser atendidos pessoalmente por médicos, em seus consultórios (BOLZANI, 2004).

A tele-educação ou educação à distância é mais uma ferramenta que tem seu uso aumentado a cada dia, cujo objetivo é a promoção do conhecimento através da tecnologia, onde os professores não estão fisicamente no mesmo ambiente que os alunos (MIGUEL \& NICOLAIO, 2008). É um processo que vem procurando promover a democratização do ensino, cujos alvos são grupos sociais que não dispõem de tempo, condições físicas ou financeiras. Dependendo do curso, as avaliações presenciais têm caráter obrigatório, conferindo ao usuário o seu devido mérito.

A questão da privacidade ainda é uma das situações que mais provoca discussões na sociedade, quando se faz uso das redes domiciliares. Quanto mais as vidas das pessoas, suas contas bancárias, carros, casas, ruas e cidades são monitoradas, digitalizadas e conectadas, mais e mais informações pessoais são disponibilizadas seja para instituições, seja para outras pessoas, implicando em invasão de privacidade e possibilidade de riscos com a segurança. Sendo assim, o simples uso de monitoramento constante ou não de algum ambiente inteligente que esteja conectado em rede pode proporcionar a possibilidade de haver algum tipo de vazamento de informação de imagem ou vídeo particular.

Existe uma linha de pesquisadores que tem colocado que a concentração e exposição de muitos equipamentos às pessoas na habitação podem levar ao isolamento do indivíduo dentro da sua casa e até mesmo dos companheiros que moram nela, afetando seu desenvolvimento social.

Apesar de haver algumas preocupações, é fato dizer que as vantagens oferecidas pela Domótica podem proporcionar um ganho considerável na qualidade de vida da sociedade.

\section{ASPECTOS AMBIENTAIS DA DOMÓTICA NO CONTEXTO HABITACIONAL}

O setor da Habitação tem sido um dos mais responsáveis por impactos ambientais, principalmente os relacionados à gestão de consumo energético. A exemplo dos prédios inteligentes, aonde a gestão de energia vem sendo colocada em primeiro plano, tanto pelo lado ambiental quanto pelo econômico, tem-se que a residência também pode seguir os mesmos passos através da aplicação da Domótica. O conforto pode ser alterado de forma imperceptível, através da gestão automatizada dos recursos naturais de um domicílio, a exemplo de algumas aplicações como a diminuição ou desligamento automático de alguma iluminação em determinado ambiente ou regulação de uso de algum aparelho de controle de temperatura. A utilização de sistemas solares e estratégias bioclimáticas associadas à Domótica também podem garantir um melhor aproveitamento da energia, como o 
acionamento de claraboias, cortinas e aquecimento de água, de acordo com as condições ambientais.

Com a entrada dos carros híbridos ou elétricos no mercado, várias residências e prédios residenciais já estão se equipando com previsão de infraestrutura própria para recarga de bateria desses novos veículos. Com o avanço dessa tecnologia, já se estuda, entre outros planos, fazer algum tipo de conexão inteligente com os carros elétricos no sentido de haver uma troca de informações entre a habitação e o veículo de forma que ele possa fornecer energia gerada para a casa, quando esta for possibilitada, e vice-versa, mediante estratégias do processo.

Com relação aos fluidos e detritos, já existem sistemas de controle que podem ser aplicados no tratamento da água, em habitações. Como exemplos de aplicações, podem ser citados:

- Utilização de luz ultravioleta ou outros meios para o tratamento de água potável e descontaminação de alimentos;

- Reutilização de água da chuva para irrigação, lavagem de carro, limpeza de pátio e descargas de vaso sanitário;

- Armazenamento e tratamento de águas cinza (provenientes de lavatórios) para reutilização.

A implantação do teletrabalho também é um fator que proporciona consequências positivas, por favorecer a redução do uso de meios de transporte nos deslocamentos casa-trabalhocasa, evitando mais emissões de carbono.

Quanto à questão do uso de energia eletromagnética e suas emissões nas redes domiciliares sem fio (wireless), pode-se dizer que a quantidade de radiação total emitida pelos sistemas de transmissão não é um fator de risco, pois seu valor é centenas de vezes menor que o sinal de um telefone celular, em conformidade com padrões internacionais, os quais a ANATEL (Agência Nacional de Telecomunicações) segue.

O conforto quando é confundido com luxo pode provocar desperdícios, devendo estes ser evitados. Os ambientes inteligentes podem, de forma imperceptível, monitorar o usuário em sua residência, interagindo e conduzindo o mesmo para boas práticas de gestão energética sem perda da qualidade de vida, diminuindo o impacto ambiental que uma residência possa trazer.

\section{ASPECTOS ECONÔMICOS DA DOMÓTICA NO CONTEXTO HABITACIONAL}

Com a redução dos custos e da complexidade de instalações domóticas graças ao aparecimento de novos padrões e tecnologia, a indústria está conseguindo aumentar a difusão e procura maior por parte dos usuários, donos de habitações que ainda não estavam dispostos a investir na construção de uma casa inteligente. O aumento da variedade de fabricantes e produtos com custo mais baixo já começa a atrair o usuário, que também começa a contar com a banda larga de acesso à Internet com tarifas mais acessíveis.

Através da interação homem - casa - tecnologia, uma enorme quantidade de aplicações comerciais são desencadeadas. Serviços de "home shopping" (compras feitas através da Internet) e "home banking" (uso de serviços bancários através da Internet) já fazem parte do cotidiano da sociedade. Os serviços e programas aplicativos emergentes devido ao uso da Domótica, assim como novas profissões, a exemplo do integrador de sistemas, também já são uma realidade (AURESIDE, 2011). Os setores de telecomunicações, telefonia, informática, saúde, serviços de entretenimento e o setor de energia são exemplos de áreas onde suas eficiências de operação em relação à habitação estão permitindo uma grande abertura de mercado frente ao usuário de um ambiente inteligente. 
A redução dos custos dos meios de transmissão e melhoria das taxas de velocidade de dados deve permitir a entrada de novos mercados e serviços ligados à TI (Tecnologia da Informação). É fato que os países desenvolvidos conseguem obter mais vantagens com esses serviços por terem condições de conectividade bem avançada, o que não acontece em países menos favorecidos. O Brasil, servindo de exemplo, possui uma população ainda pouco conectada, seja por limitações sociais (poder aquisitivo) ou por limitações de distâncias, onde o investimento de uma rede ainda não se justifica. Através do Censo 2010 (IBGE, 2010), foi constatado que apenas $39,3 \%$ dos habitantes possuíam computador em suas residências, sendo que somente $31,5 \%$ tinham acesso à Internet (na Suécia, esse valor chega a $97 \%$, praticamente o país inteiro). Esses números também poderiam ser maiores se a qualidade do serviço relativo à transmissão dos dados fosse melhor.

O mercado imobiliário também passou a ver a Domótica como uma oportunidade para aumentar seus lucros, onde as construtoras têm oferecido habitações mais "confortáveis" aos consumidores de padrão médio, através de sistemas pré-instalados. A automação residencial já começa a virar motivo de competitividade entre as construtoras, uma vez que representa a extensão do consumo da tecnologia pessoal que o usuário atual exige. Esse diferencial, que vai além do básico, representa algo em torno de $5 \%$ do valor do imóvel, em média.

No Brasil, a maioria dos equipamentos é importada de lugares como Europa e Estados Unidos. Porém, já existem empresas nacionais produzindo equipamentos de alta tecnologia em condições de competir com os produtos internacionais, prevendo a grande expansão do mercado.

A economia como um todo pode se beneficiar bastante com a Domótica. Através da sua aplicação nas casas, a sustentabilidade econômica pode ser promovida através da otimização interna da gestão de recursos como água, gás e energia elétrica, via monitoramento e prevenção de gastos e desperdícios. O investimento inicial é retornado ao passo que os custos são controlados e poupados.

Além disso, os sistemas domóticos podem trabalhar integrados a sistemas que se utilizem de fontes de energia renováveis, através do uso de geradores, como o solar ou o eólico, de forma que se possa produzir uma parcela de energia elétrica, com posterior armazenamento em algum banco de baterias para utilização futura.

O desenvolvimento e análise de métodos de utilização racional de fontes energéticas, como o exemplo da energia fotovoltaica, fez da Alemanha o seu principal mercado, onde o governo estimula a produção e investimentos pela população, apoiando uma economia sustentável. Lá, não há limites para a venda de energia para as redes concessionárias e seu valor é bastante atraente. Muitas usinas estão sendo desativadas, principalmente as nucleares graças a essa ação.

Alguns países da Europa, Ásia e Estados Unidos adotam em seus sistemas de fornecimento de energia elétrica a tecnologia chamada "Smart Grid" (Rede Elétrica Inteligente), que distribui energia elétrica usando tecnologia digital, visando à otimização do sistema através do controle e monitoramento de toda a rede de distribuição. O resultado é alcançado com melhor qualidade de energia, maior confiabilidade, maior eficiência e maior redução de custos. Isso permite que as concessionárias de energia conheçam melhor o padrão de consumo de seus clientes, fazendo uso de medidores eletrônicos inteligentes. Com isso, políticas energéticas podem ser implementadas, como a diminuição de tarifas fora de horários de pico, normalmente à noite, evitando sobrecarga de suas usinas de geração de energia. A Domótica permite atuar de forma a conduzir equipamentos, como máquinas de lavar, para trabalharem nesses períodos. Os sistemas de automação também atuam no controle do limite de energia diário ou mensal a ser utilizado numa residência, como meta, evitando o uso de equipamentos "pesados" simultaneamente. O usuário pode receber 


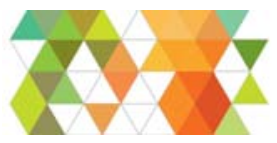

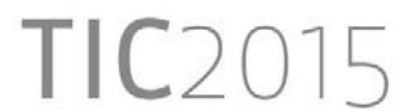

relatórios via Internet sobre os gastos diários, enviados pela concessionária de serviços (água, luz, gás, comunicações, etc.), permitindo um estudo sobre uma melhor estratégia de uso e controle do processo.

Segundo Paulino (2006), a automação do processo de medição e da leitura, com o uso de medidores inteligentes de energia elétrica, tem potencialmente uma série de vantagens:

- Combate a fraudes e furto de energia;

- Implementação de tarifas diferenciadas em função da hora de consumo (tarifa branca);

- Corte e religamento remoto de energia;

- Implementação de programas de energia pré-paga;

- Melhor acompanhamento de gastos pelo consumidor;

- Eliminação de erros e custos de processo de leitura manual.

O Brasil, através da ANEEL (Agência Nacional de Energia Elétrica), está em processo de implantação do Smart Grid. O projeto, como já citado, vai prever o uso de sistemas de geração residencial de energia em pequena escala, além de gerenciamento automático do consumo. Alguns projetos-piloto têm sido implementados a exemplo do programa chamado "Cidade do Futuro", em Sete Lagoas, Minas Gerais, com a instalação de 80 mil medidores inteligentes, e no Rio de Janeiro, nas cerca de mil residências localizadas no conjunto habitacional São Sebastião, na Zona Sul da cidade. Os novos medidores eletrônicos já estão sendo oferecidos pelas distribuidoras aos consumidores das grandes metrópoles, porém seu potencial total de utilização ainda depende de investimentos em infraestrutura por parte das concessionárias. Espera-se que até 2020 sejam instalados algo em torno de 60 milhões desses aparelhos no Brasil.

O envelhecimento da população no mundo, juntamente com o declínio da taxa de fertilidade tem sido alvo de muita preocupação para o sistema de saúde de vários países, principalmente os europeus, onde a média de idade é alta. O quadro de incapacitados vem aumentando gradativamente e doenças como Alzheimer vão aparecendo com mais intensidade na faixa da população com mais de 65 anos. O volume do suporte de familiares e auxiliares que podem ficar tomando conta dessas pessoas em casa também tem caído. Baseado em projeções de estudo de crescimento populacional, acredita-se que a demanda por cuidados públicos com a saúde vai aumentar muito, sobrecarregando o sistema e promovendo custos institucionais de previdência social e saúde cada vez maiores. Nesse sentido, os ambientes inteligentes, fazendo uso da Domótica, podem surgir como uma perspectiva de auxílio a pessoas debilitadas, mantendo as mesmas em suas casas e permitindo a prática da medicina remota. A tecnologia assistiva pode prover ao usuário monitoramento constante e remoto de sua saúde, bem como suporte em tarefas que exijam maior autonomia. Outro fator de grande aplicação é o uso da telemedicina, que pode trazer benefícios de atendimento mais rápido e eficiente, na medida em que o usuário não precisaria viajar para ser atendido por um especialista localizado em um ponto distante do seu.

\section{SUSTENTABILIDADE ATRAVÉS DO PLANEJAMENTO DE HABITAÇÕES}

Enquanto a sociedade tem se submetido a rápidas transformações decorrentes da automação, a arquitetura e o planejamento (infraestrutura) estão assimilando de forma lenta seu aparecimento.

Atualmente, muitos fatores não colaboram para que novas edificações possam estar preparadas para receber as tecnologias de forma natural. Entre eles, pode-se destacar a falta de um padrão de projeto de instalações elétricas, a qual exige uma atualização de 
conceitos que comporte não apenas o formalismo de um projeto convencional, mas todas as exigências que um imóvel moderno precisa.

Com relação às leis, a legislação brasileira ainda não adotou nenhuma regulamentação definindo as responsabilidades de avaliação e verificação da implementação de um projeto de instalação elétrica interna de uma residência por parte de um profissional devidamente habilitado, o mesmo valendo para projetos de automação, apesar de algumas capitais brasileiras possuírem legislação em análise.

No Brasil, assim como foi feito na Europa e países desenvolvidos, é preciso rever toda a legislação relacionada com as obras e instalações dentro de uma casa, desde a norma arquitetônica, a norma de ruído, de instalações elétricas, de telecomunicações, dutos, entre outras. Também se faz necessário que haja um mecanismo legal que garanta 0 cumprimento geral das normas e que possibilite uma avaliação sistemática das instalações prediais e residenciais.

É preciso que exista uma legislação técnica para definir padrões dos equipamentos usados e das automações em instalações elétricas. Atualmente existem normas para instalações elétricas, porém faltam para instalações de automação. O que existem, no Brasil, são normas para cabeamento residencial de redes, de origem norte-americana, as quais definem padrões e referências para sua correta instalação e dimensionamento.

Nos países que ajustaram recentemente suas normas técnicas, pode-se observar o exemplo da Espanha, onde construções que superem um número mínimo de metros quadrados são obrigadas pelos seus novos códigos técnicos de edificação a instalarem certa quantidade de captadores solares térmicos e placas de células fotovoltaicas para promoção de sustentabilidade, seguindo uma norma. Também ocorre, com relação à energia, certo tipo de classificação energética nas casas, de modo que seu valor agregado seja computado de acordo com sua eficiência energética, depois da sua verificação pela fiscalização de órgãos competentes.

\section{TECNOLOGIAS ENVOLVIDAS NA INFRAESTRUTURA FÍSICA E LÓGICA}

O planejamento de habitações com recursos de Domótica deve levar em consideração a utilização de tecnologias em sua infraestrutura física e lógica. Atualmente, existe uma grande diversidade tecnológica envolvendo casas inteligentes e seus sistemas domóticos, porém a falta de integração entre eles impede a universalização do seu uso.

As tecnologias envolvidas em Domótica permitem um vasto estudo relacionado à infraestrutura física e lógica, onde a maior concentração se situa nas seguintes áreas:

- Arquitetura e construção (instalações físicas e normas);

- Automação e controle (sensores e atuadores);

- Comunicação (redes de computadores);

- Processamento e reconhecimento de sinais (imagem, voz, movimento e outros);

- Sistemas operacionais (arquitetura de software);

- Teoria da informação (criação de algoritmos).

Dentre essas áreas listadas, a infraestrutura lógica, composta por programas e interfaces de comunicação exclusivas, é a mais delicada, exigindo soluções mais complexas. Surgiram muitas tecnologias interessantes, porém a existência de múltiplas alternativas, sem compatibilidade entre elas, dificultou o processo de estabelecimento dessas novas tecnologias. Para tentar resolver estes problemas, surgiram vários movimentos de normalização. Também houve a abertura de projetos, que antes eram propriedade de 
grandes empresas. A tecnologia conhecida como X10, muito usada nos Estados Unidos, passou por esse processo de abertura que, graças à expiração de sua patente, foi possível ser usada por qualquer empresa nos processos de automação residencial (NUNES, 2002).

Os movimentos de normalização ocorridos fizeram com que várias tecnologias estivessem à disposição e continuassem a disputar entre si, o que não permitiu uma padronização mais efetiva, gerando uma barreira na redução dos custos e dificuldade na escolha da melhor alternativa de tecnologia, tornando difícil um maior avanço na aplicação da Domótica.

As preocupações atuais dos vários centros de pesquisa no estabelecimento de um padrão de tecnologia estão focadas na compatibilização entre equipamentos de diversos fabricantes, não só com relação aos meios físicos e formas de transmissão dos sinais, como também na ênfase em sistemas abertos, tanto no que diz respeito a equipamentos, quanto a programas, na viabilidade econômica e tecnológica, na segurança dos usuários e dos dados e no acordo com normas e regulamentos internacionais.

O objetivo principal passa a ser uma compatibilização de equipamentos que permita, por exemplo, que o usuário compre uma televisão na loja, chegue em casa e abra a caixa, conecte a mesma na rede de energia de sua casa e ela automaticamente se comunique e interaja com o decodificador de TV a cabo, o gravador de vídeo, o sistema de som e com a Internet. Essa rede doméstica, que configura a infraestrutura lógica da habitação, utiliza programas, equipamentos pontuais e centrais para promover a ligação entre a infraestrutura física e os dispositivos a serem controlados.

Segundo Domingues (2013), dentre as tecnologias usadas em Domótica destacam-se: PLC (PowerLine Carrier), BUSLINE, wireless, e cabeamento estruturado. Os três primeiros sistemas têm como característica a pouca interferência na instalação elétrica já existente na habitação e, graças a sua facilidade de instalação, são mais indicados para estruturas já existentes e também para pequenas reformas. Para o caso de uma nova construção, os sistemas de cabeamento estruturado, com uso de centrais de automação, ainda são os mais indicados.

É importante esclarecer que as soluções de um projeto de casa inteligente podem fazer uso de diversos padrões, dependendo das necessidades e possibilidades, uma vez que haja compatibilidade entre eles e entre a central de automação, caso exista.

No que diz respeito à normalização de instalações domóticas, onde a infraestrutura física é necessária, mudanças nos projetos de instalação elétrica convencionais devem ser absorvidas e implementadas de forma a comportar as novas fiações.

\section{CONCLUSÕES}

As vantagens oferecidas pela Domótica podem proporcionar um ganho considerável na qualidade de vida da sociedade. Devido aos problemas com meio ambiente e escassez de recursos, também é importante destacar o uso das ferramentas de automação residencial para gestão do consumo de energia e monitoramento, bem como a utilização automática de fontes de energia alternativas.

Como resultado da análise do processo de integração dos diversos sistemas existentes na habitação, pode-se dizer que os principais desafios são os tecnológicos e sociais. Os desafios tecnológicos se resumem na preocupação com o estabelecimento de um padrão de tecnologia focado na compatibilização entre os equipamentos de diversos fabricantes, bem como a busca da interação através de formas mais naturais de interface do homem com a máquina, onde o computador funcionaria como uma entidade invisível, e a comunicação seria feita através de voz ou expressões gestuais. Os desafios sociais seriam a falta de consciência e cultura da população quanto à verdadeira importância da Domótica para o 
auxílio diário aos moradores de uma residência.

Com a entrada de sistemas de distribuição elétrica à base de Smart Grid, vários serviços poderão se transformar em soluções que poderão passar a pertencer à rotina de uma habitação, exigindo cada vez mais as mudanças de infraestrutura para sua implementação.

A possibilidade de um aumento da taxa de transmissão das redes de comunicação de dados e facilidades de acesso através de uma melhor distribuição das mesmas deve favorecer a utilização e desenvolvimento da Domótica de forma considerável.

A falta de mão de obra qualificada também é um desafio a ser vencido. O surgimento de novas interfaces obriga os profissionais da área, no caso os projetistas, a terem que desenvolver uma visão sistêmica e integrada. Algumas universidades em várias partes do mundo já estão adequando seus cursos para formação de profissionais com essa especialização.

A Domótica se torna, a cada dia, primordial para a integração de dois importantes contingentes da sociedade: idosos e incapacitados, que somados podem corresponder a $10 \%$ da população brasileira, porcentagem essa que tende a aumentar graças ao envelhecimento populacional que é uma previsão das pesquisas. Por meio da Domótica, barreiras podem ser eliminadas quanto a questões como emprego, saúde, educação, acessibilidade, conforto e qualidade de vida, através da promoção da independência, privacidade, dignidade e integração social.

Finalizando, uma habitação suficientemente integrada, para assistir os usuários de forma consciente e confortável através da ajuda da tecnologia, onde todos os equipamentos eletroeletrônicos estejam interconectados, formando uma rede que possa se comunicar entre eles e com o usuário de forma interativa e proativa, ainda está longe de se tornar realidade, porém as expectativas mostram que esse conceito de habitação pode se tornar realmente o padrão do futuro.

\section{REFERÊNCIAS}

ALDRICH, F. Smart Homes: Past, Present and Future. In: HARPER, R. (Ed.). Inside the Smart Home. Springer Verlag, Reino Unido, 2003. p.17-36.

AURESIDE. Associação Brasileira de Automação Residencial. Disponível em: <http://www.aureside.org.br>. Acesso em: 13 nov. 2011.

BOLZANI, C. A. M. Residências Inteligentes: um curso de domótica. 1a. ed., São Paulo: Editora Livraria da Física, 2004. 332p.

DOMINGUES, R. G. A Domótica como Tendência na Habitação: Aplicação em Habitações de Interesse Social com Suporte aos Idosos e Incapacitados. Dissertação (Mestrado em Engenharia Urbana), Programa de Engenharia Urbana, Escola Politécnica, Universidade Federal do Rio de Janeiro, Rio de Janeiro, 2013. 147p.

ELOY, S. et al. Utilização de Domótica na Estratégia de Sustentabilidade Social e Ambiental. In: CONGRESSO INTERNACIONAL DE HABITAÇÃO NO ESPAÇO LUSÓFONO, 2010, Lisboa. Anais... Instituto Universitário de Lisboa, Lisboa, 2010. p.22-24.

FORTY, A. Objetos de Desejo: Design e Sociedade desde 1750. Tradução: Pedro Maia Soares, São Paulo: Editora Cosac Naify, 1986. 352p.

INSTITUTO BRASILEIRO DE GEOGRAFIA E ESTATÍSTICA. Síntese de indicadores sociais: uma análise das condições de vida da população brasileira, 2010. IBGE, Coordenação de População e Indicadores Sociais. Rio de Janeiro: IBGE, 2010. 321p. 
LARCHER, J. V. M. Diretrizes visando a melhoria de projetos e soluções construtivas na expansão de habitações de interesse social. Dissertação (Mestrado em Construção Civil), Universidade Federal do Paraná, Curitiba, 2005. 160p.

MIGUEL, L.; NICOLAIO, K. A democratização do ensino por meio da educação à distância. TCC (Curso de Pedagogia), Faculdade Internacional de Curitiba (FACINTER), Paraná, 2008. 24p.

NUNES, R. Análise comparativa de tecnologias para Domótica. In: III JORNADA DE ENGENHARIA DE AUTOMAÇÃO, CONTROLO E INSTRUMENTAÇÃO, 2002, Setúbal. Anais... JEACI, 2002.

PAULINO, C. A. Estudo de tecnologias aplicáveis à automação da medição de energia elétrica residencial visando a minimização de perdas. Dissertação (Mestrado em Engenharia Elétrica), Escola Politécnica da Universidade de São Paulo, São Paulo, 2006. 101p.

SATPATHY, L. Smart housing: Technology to aid aging in place - New opportunities and challenges. Dissertação (Mestrado em Arquitetura), Universidade do Estado de Mississippi, Estados Unidos, 2006. 173p.

UN DEPARTMENT OF ECONOMIC AND SOCIAL AFFAIRS - Population Division. World Population Prospects, 2010. Disponível em: <http://esa.un.org/unpd/wpp/index.htm>. Acesso em: 12 jul. 2012.

YUSUPOV, R. M.; RONZHIN, A. L. From Smart Devices to Smart Space. Herald of the Russian Academy of Sciences, v.80, n.1, p.63-68. Rússia: Pleiades Publishing, 2010. 